\title{
BMJ Open Impact of the Southwark and Lambeth Integrated Care Older People's Programme on hospital utilisation and costs: controlled time series and cost- consequence analysis
}

\author{
Josephine Exley, ${ }^{1}$ Gary A Abel, ${ }^{2}$ José-Luis Fernandez, ${ }^{3}$ Emma Pitchforth, ${ }^{2}$ \\ Silvia Mendonca, ${ }^{4}$ Miaoqing Yang, ${ }^{1}$ Martin Roland, ${ }^{4}$ Alistair McGuire ${ }^{5}$
}

To cite: Exley J, Abel GA, Fernandez J-L, et al. Impact of the Southwark and Lambeth Integrated Care Older People's Programme on hospital utilisation and costs: controlled time series and cost-consequence analysis. BMJ Open 2019;9:e024220. doi:10.1136/ bmjopen-2018-024220

- Prepublication history and additional material for this paper are available online. To view these files, please visit the journal online (http://dx.doi org/10.1136/bmjopen-2018024220).

Received 15 May 2018 Revised 1 September 2018 Accepted 29 November 2018

Check for updates

(C) Author(s) (or their employer(s)) 2019. Re-use permitted under CC BY-NC. No commercial re-use. See rights and permissions. Published by BMJ.

For numbered affiliations see end of article.

Correspondence to Professor Martin Roland; mr108@cam.ac.uk

\section{ABSTRACT}

Objectives To estimate the impact on hospital utilisation and costs of a multi-faceted primary care intervention for older people identified as being at risk of avoidable hospitalisation.

Design Observational study: controlled time series analysis and estimation of costs and cost consequences of the Programme. General practitioner (GP)'s practice level data were analysed from 2009 to 2016 (intervention operated from 2012 to 2016). Mixed-effect Poisson regression models of hospital utilisation included comparisons with control practices and background trends in addition to within-practice comparisons. Cost estimation used standard tariff values.

Setting 94 practices in Southwark and Lambeth and 263 control practices from other parts of England.

Main outcome measures Hospital utilisation: emergency department attendance, emergency admissions, emergency admissions for ambulatory sensitive conditions, outpatient attendance, elective admission and length of stay.

Results By the fourth year of the Programme, there were reductions in accident and emergency (A\&E) attendance (rate ratio $0.944,95 \% \mathrm{Cl} 0.913$ to 0.976 ), outpatient attendances (rate ratio $0.938,95 \% \mathrm{Cl} 0.902$ to 0.975 ) and elective admissions (rate ratio $0.921,95 \% \mathrm{Cl} 0.908$ to 0.935 ) but there was no evidence of reduced emergency admissions. The costs of the Programme were £149 per resident aged 65 and above but savings in hospital costs were only £86 per resident aged 65 and above, equivalent to a net increase in health service expenditure of $£ 64$ per resident though the Programme was nearly cost neutral if set-up costs were excluded. Holistic assessments carried out by GPs and consequent Integrated Care Management (ICM) plans were associated with increases in elective activity and costs; £126 increase in outpatient attendance and $£ 936$ in elective admission costs per holistic assessment carried out, and $£ 576$ increase in outpatient and $£ 5858$ in elective admission costs per patient receiving ICM.

Conclusions The Older People's Programme was not cost saving. Some aspects of the Programme were associated with increased costs of elective care, possibly through the identification of unmet need.

\section{Strengths and limitations of this study}

- The analysis covered a 7-year period: 3 years prior to and 4 years following the start of the Programme including a set of matched controls from other parts of England.

- We examined within-practice changes using random effects models. We analysed rates of hospital utilisation across different age and gender groups, to allow for potential changes in the age profile of the population over the course of the study period. Models also adjusted for individual practice characteristics and the effect of the time of year (seasonality).

- For two specific components of the intervention (holistic assessments and Integrated Care Management), we were able to look for a 'dose-effect' to see whether the delivery of these elements appeared to have independent effects on hospital utilisation

- Sensitivity analyses, excluding small numbers of practices with unusually high rates of admission or mean length of stay, did not alter our conclusion.

- The analysis focused on measures of secondary care use; however, the intervention might have had an effect that went beyond these outcomes.

\section{INTRODUCTION}

It is widely recognised that care of elderly people falls short of the ideal. Reasons for this include failure to identify developing medical problems and failure to provide well-coordinated care for people with multiple complex problems, both of which may lead to avoidable emergency admissions. ${ }^{12} \mathrm{~A}$ wide range of initiatives have been developed in recent years but most include, in some form, identifying patients in need of more intensive or coordinated management and then intervening with proactive packages of care designed to maximise patients' abilities to self-manage their conditions and anticipate 
preventable deteriorations in health. Risk profiling and intensive case management form common parts of these approaches. $^{3-7}$

The conceptual basis behind case management interventions lies in the Chronic Care Model, which includes using clinical information systems to plan patient care and redesigning the delivery of care to meet the needs of patients with chronic illness. ${ }^{8}$ Payers have focused on these elements of the model to identify patients with high healthcare costs, hoping that better targeted and coordinated care will improve care and reduce costs though often with a focus on costs as the primary outcome. Uncontrolled studies of healthcare utilisation in this group often show reduction in utilisation, which may simply result from regression to the mean ${ }^{9}$ and systematic reviews of rigorous evaluations of case management and interventions to integrate or coordinate care have, on the whole, shown much smaller effects. ${ }^{61011}$ One problem is whether case management interventions should target the highest risk group who are likely to show the greatest impact on individuals but unlikely to show much impact on healthcare costs overall. ${ }^{9}$ The Southwark and Lambeth Integrated Care (SLIC) Older People's Programme ('the Programme') reported in this paper took a population-wide approach. The Programme originally intended to carry out holistic assessments (HAs) in general practice with half of all residents aged 65 and above in the two south London boroughs, combined with a range of primary and secondary care interventions and targeted case management for those identified as at risk.

The Programme was set up in 2012 to maximise the health and independence of older people and minimise avoidable hospital utilisation. ${ }^{12}$ SLIC brought together general practitioners (GPs), hospitals and local authorities to redesign services and provide better integrated care for people aged 65 and above. The Programme consisted of many different projects, which addressed a range of aspects of health and social care, summarised in box 1. Alongside the projects listed, the Programme aimed to support the development of the wider system enablers of change such as IT infrastructure.

A key element of the Programme was the introduction of a screening tool, HAs, to proactively identify health and social care needs of people aged 65 and above within General Practice in the two boroughs and then put care plans in place to address those needs that might otherwise have led to avoidable secondary care use. Each HA included assessment of the patient's physical health, mental health and social care needs, as well as wider social aspects of daily living (eg, benefits and housing). Patients identified as requiring additional support were allocated for Integrated Care Management (ICM), conducted by a dedicated member of staff from a local care provider. Care managers varied from patient to patient. For example, a patient with complex medical needs may be care managed by their named GP while someone requiring more non-medical support and service coordination could be care managed by another trained professional within the
Box 1 Summary of the main elements of the Southwark and Lambeth Integrated Care Older People's Programme

Better proactive identification of need and interventions to avoid crisis

- Holistic assessments (HAs): a proactive and HA of need, for older people, undertaken by general practitioner (GP) practices.

- Integrated Care Management plans for older people identified as 'at risk' in holistic assessments: additional named support for care coordination/navigation or wider care planning.

- Community-based multi-disciplinary team meetings: these include hospital, community and social care staff who support care managers and GPs with challenging care management or system blockages.

- Redesigned clinical pathways including for falls, infections, nutrition and dementia.

\section{An alternative acute response}

- Consultant 'hotline' and 'hot clinic': direct access to specialist hospital phone line and rapid access clinics for community staff and GPs to support immediate action planning and admission avoidance.

- Enhanced Rapid Response: community-based therapy, nursing and social care to support people to stay in their home and prevent an admission to hospital or to support them to be discharged from hospital earlier in their stay.

- @home: multi-disciplinary team providing acute clinical care at home which otherwise would be carried out in hospital.

Maximising independence before long-term care is finalised

- Simplified discharge processes: designing options for a unified point of access for community and social care services at the point of discharge and new models to improve the discharge process for patients returning home or to a care home.

practice team. Where patients presented with complex problems or where system blockages were present, cases could be presented and discussed at a local Community Multi-Disciplinary Team (CMDT).

The intention was for GPs to undertake an HA with half of all their patients aged 65 and above. However, from the outset, the Programme experienced difficulties in delivering the anticipated activity targets for HAs, ICM and CMDT. Low levels of activity were attributed to a number of reasons; for example, some primary care staff felt interventions were imposed on them and that they lacked time to engage with the Programme. ${ }^{13}$ In 2014 , recovery plans were put in place, the HAs were reviewed and targets were revised. This resulted in the eligible population for HAs being changed to all people aged 80 or above and people aged above 65 who were either housebound or had not been seen by a GP for 15 months. In 2015, this bundle of projects was expanded to include Care Navigation and Locality Geriatricians.

This evaluation was commissioned to run in parallel with the Programme from August 2012 until the end of the Programme in March 2016, with the principle aim of examining changes in hospital utilisation by people aged 65 and above registered with GPs in Southwark and Lambeth following implementation of the Programme. 
In most respects, it was not possible to single out which elements of the Programme were more or less successful in achieving the wide range of aims originally set out by the SLIC partners. However, we were able to estimate the overall effect of the Programme on hospital admissions and length of stay and, because of the particular way the Programme developed, we were able to isolate the effects of HAs and ICM plans for those identified as 'at risk'.

\section{METHODS}

Using Hospital Episode Statistics (HES) ${ }^{14}$ provided by National Health Service (NHS) Digital, which contains details of all admissions, outpatient appointments and accident and emergency (A\&E) attendance at NHS hospitals in England, we considered five outcome measures of hospital utilisation for people aged 65 and above registered at a GP practice in Southwark and Lambeth:

1. A\&E attendance.

2. emergency hospital admissions.

3. emergency hospital admissions for patients with admissions for 'ambulatory care sensitive' (ACS) conditions recorded as one of the diagnoses on discharge. ${ }^{15}$ ACS conditions are those for which, in principle, crises leading to emergency admissions that might be prevented by improved care in the community.

4. outpatient attendance.

5. elective hospital admissions.

A series of analyses were performed using a Poisson-regression-based approach that allowed for the longitudinal and clustered nature of the data. Data were analysed for 3 years prior to and 4years following the start of the Programme (second quarter of 2009 to first quarter of 2016) in 5-year age-band by gender strata. By analysing rates of hospital utilisation across different age and gender groups, we allow for potential changes in the age profile of the population over the course of the study period. In addition to age and gender, models also adjusted individual practice characteristics and for the effect of the time of year (seasonality). Further details of the methods and full regression results are shown in the online supplementary appendix. We carried out sensitivity analyses excluding small numbers of practices with unusually high rates of admission or mean length of stay, but none of these made a material difference to our conclusions and they are not reported here. In addition to these outcomes, we also analysed length of stay and found no effect of any of the interventions (full results of the length of stay analysis are in online supplementary tables A1 and A2).

In our first analysis, we compared trends in these practices with those in a matched group of control practices from other parts of England using a 'genetic' matching algorithm. ${ }^{16}$ Originally, control practices were matched with a ratio of five controls for every intervention practice with replacement, meaning that control practices could appear more than once in the comparison dataset. However, due to the extended period of analysis, we found a significant number of practices had closed, meaning that it would be impossible to maintain exact matching. As a consequence, we felt the advantage of including duplicate records for some practices was minimal and only a single copy of data from each practice was used. Practices were matched on basis of list size, the proportion of patients registered with the practice above the age of 65 , the proportion of patients registered with the practice above the age of 80 , the proportion of male patients, the mean years since qualification of GPs, the number of patients per full-time equivalent GP (a measure of workload), the proportion of full-time equivalent GPs made up by male GPs, practice deprivation score and the proportion of the practice population describing themselves as white. In addition, control practices were matched on baseline admissions/attendance and the rate of change of those admissions/attendance at baseline, in this case not including A\&E attendance. Further details of the matching are given in the online supplementary appendix.

A second set of analyses took account of the variation in the level of activity that occurred between practices in Southwark and Lambeth for two key elements of the Programme: HAs and ICM. Since some practices implemented these more rapidly and more comprehensively than others, we were able to look for a 'dose-effect' to see whether the delivery of these two specific elements of the Programme appeared to have independent effects on hospital utilisation. We used the percentage of patients aged 65 and above who had received one of the interventions at each of 13 3-month postintervention time points as a measure of the 'dose' of intervention in that practice at that time (using the cumulative total percentage at each time point). For these analyses, we conducted two separate regressions: one estimating the effect of increasing HAs and ICM in comparison to our control group of practices and the second restricting the analysis of increasing HAs and ICM to practices in Southwark and Lambeth. The restricted analysis allows for practices in Lambeth and Southwark being 'special' in some way, for example, particular ethnic profiles, which were different from other parts of the country. We report the latter set of results in the main paper as more reliably estimating differences in primary care activity between practices that have performed more HAs/ICM and those practices performing fewer or none while the comparison with control practices may also reflect a more general effect of the Programme (eg, changes to budgets, changes in other services including secondary care provision). For completeness, we present both sets of results in the online supplementary appendix, with the models described in more detail in online supplementary appendix box A1.

For the economic analysis, we drew data on the costs of the SLIC Programme from a Kings College London report. ${ }^{17}$ This estimated the costs of the whole Programme over the period at $£ 7.4$ million of which $£ 2.9$ million were infrastructure or 'enabling' costs associated with the initial establishment of the Programme. Averaging these 
costs across all residents aged 65 and above in Southwark and Lambeth gives SLIC implementation costs per older person of $£ 149$ across the life of the Programme, or $£ 91$ excluding infrastructure/enabling costs. Hospital utilisation costs were taken from the NHS Improvement Tariff Payment System ${ }^{18}$ and the Personal Social Services Research Unit cost book ${ }^{19}$. These give average, upper and lower bounds for NHS payments for A\&E attendance, emergency and elective admissions and outpatient attendance (see online supplementary appendix, table A3). In the paper, we report the results for estimated average costs incurred, with sensitivity analyses using the upper and lower bounds for both cost and potential impact on hospital utilisation reported in online supplementary appendix table A4. We only estimated costs where there were statistically significant effects on the outcomes and we did not carry out this analysis for ACS conditions to avoid double counting.

We used Stata V.15 for data management and all models were run in SAS V.9.4.

Strengthening the Reporting of Observational Studies in Epidemiology and Consolidated Health Economic Evaluation Reporting Standards checklists are included as online supplementary files.

\section{Patient and public involvement}

The analyses of secondary data carried out in this study were specified in the final protocol agreed with the funder. SLIC had its own patient representative group and a member of this group was present at meetings of the SLIC Evaluation Steering Group which reviewed interim findings. There was no other direct patient or public involvement in developing or reporting on the analyses reported in this paper.

\section{RESULTS}

We analysed data from 357 practices, including 94 practices in Southwark and Lambeth and 263 matched control practices. For reasons described above, some control practices were selected more than once in the matching procedure: of the matched practices, $164(62 \%)$ are practices that were selected only once. In total, $61(23 \%)$ practices were selected twice and the remaining $38(15 \%)$ of control practices are made up of practices that appear three or more times.

\section{Quality of matching}

We examined the extent to which the intervention and control practices were matched for baseline characteristics, the results of which are shown graphically in online supplementary appendix figures A1-A17. The variables showing substantial departures from the national profile are the percentage of patients who are above 65 (online supplementary appendix figure A10), above 85 (online supplementary appendix figure A11), the practice deprivation score (online supplementary appendix figure A13) and patients who described themselves as white (online supplementary appendix figure A14). In particular, we see that intervention practices tend to have fewer old patients compared with England and are on average located in more deprived areas (ie, their Index of Multiple Deprivation (IMD) score is higher). In general, the matching has done a reasonable job of reproducing the distribution of matching variables in the intervention practices, even for those variables where substantial departures are seen from the national distribution. However, some small, and statistically significant, deviations remain. As described above, we further adjusted for practice characteristics in the analysis to isolate so far as possible the effect of the intervention.

\section{Overall impact of the SLIC Programme on hospital utilisation} Table 1 shows the overall effect of the Programme on the five hospital utilisation outcomes by the end of the Programme in 2016. A more detailed year-by-year breakdown of the results is shown in online supplementary appendix tables A5-A14).

Table 1 Hospital utilisation: comparison with control practices by year 4 of the SLIC Programme

\begin{tabular}{|c|c|c|c|c|}
\hline Outcome & Rate ratio* $(95 \% \mathrm{Cl})$ & $P$ value & $\begin{array}{l}\text { Observed rate for SLIC } \\
\text { practices per } 1000 \\
\text { patients per year }\end{array}$ & $\begin{array}{l}\text { Expected rate in the } \\
\text { absence of SLIC } \\
\text { intervention per } 1000 \\
\text { patients per year }\end{array}$ \\
\hline A\&E attendance & 0.944 (0.913 to 0.976$)$ & 0.001 & 144 & 153 \\
\hline Emergency admissions & 1.011 (0.971 to 1.052$)$ & 0.600 & NS† & NS \\
\hline $\begin{array}{l}\text { Emergency admissions } \\
\text { for ACSCs }\end{array}$ & $1.073(1.004$ to 1.147$)$ & 0.037 & 20 & 19 \\
\hline Elective admissions & 0.938 (0.902 to 0.975$)$ & 0.001 & 153 & 164 \\
\hline Outpatient attendance & 0.921 (0.908 to 0.935$)$ & 0.001 & 1220 & 1324 \\
\hline
\end{tabular}

${ }^{*}$ Rate ratios for model 1 represent the relative change in the rate of admission compared to what would have been expected in the absence of SLIC activity. A rate ratio of 1 indicates no change, whereas a rate ratio greater than 1 represents an increase, and a rate ratio less than 1 represents a decrease, in admissions.

†No significant change.

A\&E, accident and emergency; ACSCs, ambulatory care sensitive conditions; SLIC, Southwark and Lambeth Integrated Care. 
Table 2 Effect of holistic assessments and additional hospital costs

\begin{tabular}{|c|c|c|c|c|}
\hline Outcome & Rate ratio* $(95 \% \mathrm{Cl})$ & $P$ value & $\begin{array}{l}\text { Expected change } \\
\text { per } 10,000 \text { holistic } \\
\text { assessments }\end{array}$ & $\begin{array}{l}\text { Additional hospital } \\
\text { costs incurred per } \\
\text { holistic assessment }\end{array}$ \\
\hline A\&E attendance & 1.000 (0.999 to 1.001$)$ & 0.760 & NSt & $£ 0$ \\
\hline Emergency admissions & 1.001 (1.000 to 1.002$)$ & 0.201 & NS & $£ 0$ \\
\hline $\begin{array}{l}\text { Emergency admissions } \\
\text { for ACSCs }\end{array}$ & 1.001 (0.998 to 1.003$)$ & 0.516 & NS & $£ 0$ \\
\hline Outpatient attendance & 1.002 (1.001 to 1.002$)$ & $<0.001$ & 9149 & $£ 126$ \\
\hline
\end{tabular}

${ }^{*}$ Rate ratios for model 1 represent the relative change in the rate of admission compared to what would have been expected in the absence of SLIC activity. A rate ratio of 1 indicates no change, whereas a rate ratio greater than 1 represents an increase, and a rate ratio less than 1 represents a decrease, in admissions.

†No significant change.

A\&E, accident and emergency; ACSCs, ambulatory care sensitive conditions.

The Programme was associated with a reduction in A\&E attendance by patients aged 65 and above compared with that expected based on control practices and background trends. This reduction was only evident by the end of the Programme: more detailed year-by-year results (online supplementary appendix, table A5) show a small initial rise in attendance followed by no change in the middle of the Programme and a reduction by the fourth year. Compared with that expected based on control practices and background trends, there was no evidence of a reduction in emergency admissions (a key aim of the Programme) in any of the 4 years, but a small rise in emergency admissions for ACS conditions, a rise that was evident in all 4 years of the Programme (online supplementary appendix table A9). There were significant reductions in attendance at outpatients and in elective admissions, neither of which were stated aims of the Programme.

Over the 4years of the Programme, there was a net reduction in hospital costs of $£ 86$ per Southwark and Lambeth resident aged 65 or above. Against an overall cost of implementing the SLIC Programme of $£ 149$, this represents a net increase in cost to the NHS of $£ 64$ per resident. If the infrastructure/enabling costs are removed (as these might not be ongoing once the Programme was established), then the net saving in hospital costs (£86) is very close to the costs of the Programme (£91) and sensitivity analyses (online supplementary table A14) using lower bounds for costs, and effect on hospitalisation shows the potential for the Programme to be cost saving.

\section{Impact of HAs and ICM on hospital utilisation}

At the end of the evaluation in March 2016, 26.9\% of the population aged 65 and above had received a HAs, ranging from $0 \%$ to $94.1 \%$ of the population aged 65 and above in individual general practices. $3.5 \%$ of the population aged 65 and above had been referred for ICM, ranging from $0 \%$ to $18.3 \%$ of the population aged 65 and above in individual general practices.

Table 2 shows the average change in the rate of hospital utilisation for the six outcomes for a $1 \%$ increase in the proportion of the population aged 65 and above receiving HAs. The models account for individual practice pre-intervention trends, seasonality and the full

Table 3 Effect of Integrated Care Management and additional hospital costs

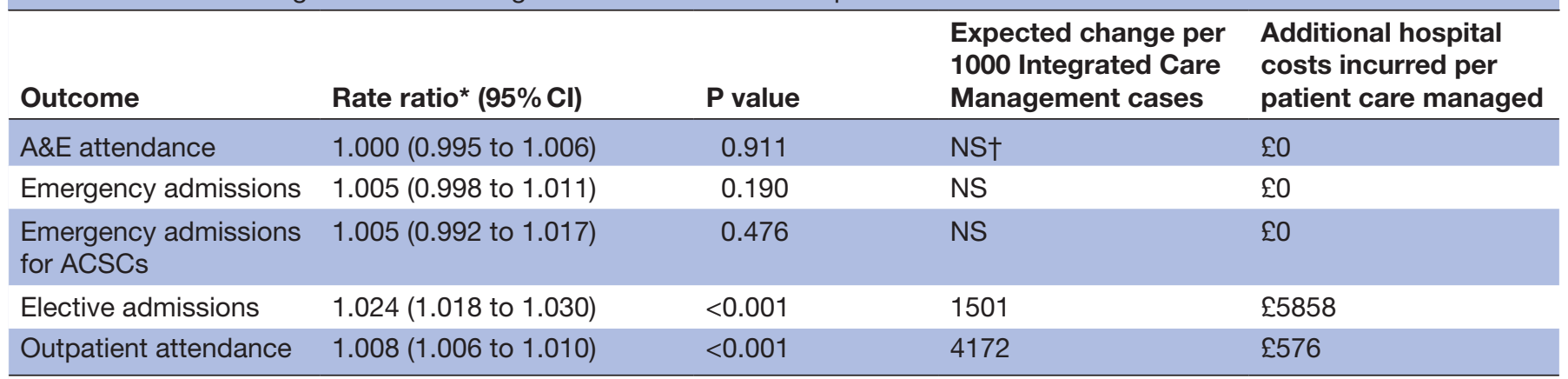

*Rate ratios for model 1 represent the relative change in the rate of admission compared to what would have been expected in the absence of SLIC activity. A rate ratio of 1 indicates no change, whereas a rate ratio greater than 1 represents an increase, and a rate ratio less than 1 represents a decrease, in admissions.

†No significant change.

A\&E, accident and emergency; ACSCs, ambulatory care sensitive conditions. 
range of individual practice characteristics. Table 3 shows the similar analyses, this time with ICM as the outcome. In each case, we report the additional secondary care costs incurred association with the increased primary care activities.

These results show no changes in relation to any of the outcomes with the exception of outpatient attendance and elective admissions for which there was a substantial, and unanticipated, increase in hospital utilisation and consequently in secondary care costs associated with the delivery of HAs and ICM.

\section{DISCUSSION}

SLIC's Older People Programme was one of a number of NHS initiatives designed to improve care for older people but had an important additional objective of reducing costs. The rationale for the commonly held belief that better integrated care will reduce costs is that poorly coordinated care may lead to unnecessary healthcare expenditures, for example through avoidable emergency admissions to hospital.

The principal finding of this evaluation was that compared with expected trends, based on data from matched control practices and background trends, NHS costs increased rather than decreased over the course of the Programme although it might have been cost neutral if set-up and infrastructure costs had been excluded. A key aim of the Programme to reduce emergency admissions was not achieved though there was some evidence of reduced A\&E attendance towards the end of the Programme. For two key elements of the Programme, HAs and ICM for patients identified as being at higher risk, there was clear evidence that despite an overall reduction in volume of elective care compared with what would have been expected the two interventions increased both outpatient attendances and elective admissions and, as a result, led to significant increases in NHS costs. Although we were not able to determine the nature of the conditions giving rise to this increased secondary care activity, based on the observed increase in elective admissions and outpatient attendance, it seems reasonable to speculate that this was due to the identification of unmet needs as a result of these enhanced primary care activities that were central to the SLIC Programme. By accounting for within practice changes, our analysis implies that if the number of HAs and ICM conducted within a practice was being driven by patient need, then the number of planned admissions was accelerating in practices with the greatest need. If this is true, it is an important message; programmes aimed at integration may not always be cost reducing. In particular, it would be of value to understand whether the increased elective care was to receive interventions that are likely to have a major effect on quality of life or whether these were likely to be for interventions of lower value.

There are a number of limitations to the study. This type of observational analysis is always potentially biased by unknown or unmeasured confounders. While it is standard to allow for confounders by using external controls, many areas of England were adopting some sort of initiative to better coordinate care. We deliberately avoided some areas with well-known integrated care schemes when selecting controls (eg, North West London) but problems inevitably remain in identifying matched controls. We addressed this by looking at within-practice changes using random effects models, using a broadly similar set of practices and further adjusting for the practice characteristics, which were included in the matching process. Using this approach, we expect our models to be robust to any systematic differences between control practices and SLIC practices with the exception of the Programme that was the focus of the evaluation. Furthermore, our dose-response, based on the intensity of HAs and ICM, restricted the analysis to practices within Southwark and Lambeth where the level of HA and ICM activity was known. This analysis effectively treats all Southwark and Lambeth practices as controls for each other and overcomes some of the limitations associated with identifying controls.

In line with the specified aims of the Programme, the analysis focused on measures of secondary care use. However, the intervention might have had an effect that went beyond these outcomes such as improved patient outcomes or experience of care. Future studies should seek to look at whether interventions that aimed to improve coordination of care resulted in gains beyond direct costs of hospital use and whether this differed for patients with different health status.

A further weakness is that, with the exception of HAs and ICM plans, we were unable to relate the changes we found to the wide range of initiatives undertaken by SLIC, some of which developed more slowly than was originally intended. ${ }^{13}$ The observed difference in impact on elective care between the overall effect of the Programme and the dose-response analysis suggest that projects other than HAs and ICM may be responsible for the overall reductions in elective care observed. Therefore, in order to fully interpret the findings, it would be important to understand which of the original plans (eg, as shown in box 1) were actually implemented and to what time-scale and how comprehensively they were implemented. Full information on this was not available to us and would be needed to draw adequate conclusions about whether individual facets of the intervention 'worked' or did not.

Our findings are consistent with several previous studies. For example, a systematic review of interventions to improve coordination in primary care found that around half of studies demonstrated a positive outcome for either health or patient experience, but less than a fifth found any reduction in health service costs. ${ }^{20}$ Some studies have found that interventions of a similar type (risk profiling and case management) increase costs initially but that cost savings may accrue after a period of time,${ }^{21}$ and we found evidence that reductions in some secondary care activities appeared towards the end of the 4-year Programme. A more recent systematic review 
of case management of at-risk patients in primary care found no evidence that these interventions produced a reduction in either health service costs or utilisation, though the authors reported 'very small' but significant improvements in self-rated health associated with the introduction of case management. ${ }^{6}$ It may be that some of the negative evaluations of previous case management programmes result from insufficient time for the evaluations to bed in. These may take more time than payers anticipate, especially where changes to working practices and culture are required. ${ }^{22}$

The principal implication of these findings for clinicians, NHS managers and policy-makers is that interventions, which aim to improve coordination of care, especially if they use some form of population case finding, should focus on improving care rather than reducing costs. Such programmes may identify unmet need and hence lead to potentially appropriate increased use of specialist care. Future studies should focus on improvements in quality of care and not treat the costs of care as being the main outcome of importance.

\section{Author affiliations}

${ }^{1}$ Cambridge Centre for Health Services Research, RAND Europe, Cambridge, Cambridgeshire, UK

${ }^{2}$ University of Exeter Medical School, Exeter, UK

${ }^{3}$ Personal Social Services Research Unit, London School of Economics, London, UK ${ }^{4}$ Cambridge Centre for Health Services Research, University of Cambridge,

Cambridge, UK

${ }^{5}$ Department of Health Policy, London School of Economics, London, UK

Acknowledgements The authors would like to thank those individuals from the SLIC sponsor board and members of the evaluation steering committee for their support throughout this evaluation, in particular Merav Dover, Moira McGrath and John Moxham. We gratefully acknowledge the helpful and insightful comments from Quality Assurance reviewers for our final report to the funder, Catherine Saunders, Jon Sussex and Alex Sutherland. They provided a critical review of project outputs and valuable advice.

Contributors All authors (JE, GA, JF, EP, SM, MY, MR and AM) contributed to the conception or design of the work, the acquisition, analysis or interpretation of the data. GA, JE and SM led on the statistical analysis. AM and JF led on the economic analysis. MR is the guarantor of the study. All authors (JE, GA, JF, EP, SM, MY, $M R$ and $A M$ ) were involved in drafting and commenting on the paper and have approved the final version.

Funding The study was funded by the Guy's and St Thomas' charity, no STR 120201. The study was sponsored by RAND Europe.

Competing interests No support from any organisation for the submitted work apart from the research grant from Guy's and St Thomas's charity

Patient consent for publication Not required.

Ethics approval Ethical approval was not required for the study as it used standard Hospital Episode Statistics extracts from NHS Digital, fully anonymised and with no sensitive fields.

Provenance and peer review Not commissioned; externally peer reviewed.

Data sharing statement № additional data are available.

Open access This is an open access article distributed in accordance with the Creative Commons Attribution Non Commercial (CC BY-NC 4.0) license, which permits others to distribute, remix, adapt, build upon this work non-commercially, and license their derivative works on different terms, provided the original work is properly cited, appropriate credit is given, any changes made indicated, and the use is non-commercial. See: http://creativecommons.org/licenses/by-nc/4.0/.

\section{REFERENCES}

1. Imison C, Poteliakhoff E, Thompson J. Older people and emergency bed use: Exploring variation. Kings Fund. 2012. www.kingsfund.org. uk/sites/default/files/field/field_publication_file/older-people-andemergency-bed-use-aug-2012.pdf

2. Tian Y, Dixon A, Gao H. Data briefing: emergency hospital admissions for ambulatory care-sensitive conditions. London: The King's Fund, 2012. www.kingsfund.org.uk/sites/default/files/field/field publication_file/data-briefing-emergency-hospital-admissions-forambulatory-care-sensitive-conditions-apr-2012.pdf

3. Georghiou T, Steventon A, Billings J, et al. Predictive risk and health care: an overview. Evidence for better health care. Nuffield Trust. 2011. https://www.nuffieldtrust.org.uk/files/2017-01/predictive-riskand-health-care-overview-web-final.pdf

4. Wallace E, Stuart E, Vaughan N, et al. Risk prediction models to predict emergency hospital admission in community-dwelling adults: a systematic review. Med Care 2014;52:751-65.

5. Ross S, Curry N, Goodwin N. Case management: What is it and how can it best be implemented? Kings Fund. London. 2011 www. kingsfund.org.uk/sites/files/kf/Case-Management-paper-The-KingsFund-Paper-November-2011_0.pdf

6. Stokes J, Panagioti M, Alam R, et al. Effectiveness of case management for 'at risk' patients in primary care: a systematic review and meta-analysis. PLoS One 2015;10:e0132340.

7. Nuffield Trust. Choosing a predictive risk model: a guide for commissioners in England. London: Nuffield Trust, 2011. www. nuffieldtrust.org.uk/files/2017-01/choosing-predictive-risk-modelguide-for-commissioners-web-final.pdf

8. Wagner EH, Austin BT, Von Korff M. Organizing care for patients with chronic illness. Milbank Q 1996;74:511-44.

9. Roland M, Abel G. Reducing emergency admissions: are we on the right track? BMJ 2012;345:e6017.

10. Baker JM, Grant RW, Gopalan A. A systematic review of care management interventions targeting multimorbidity and high care utilization. BMC Health Serv Res 2018;18:65.

11. Baxter S, Johnson M, Chambers $D$, et al. The effects of integrated care: a systematic review of UK and international evidence. BMC Health Serv Res 2018;18:350.

12. Southwark and Lambeth Integrated Care. http://www.lambethccg. nhs.uk/our-plans/our-programmes/slic/Pages/slic.aspx

13. Ross S, Fitzsimons B. Final report of the evaluation of the process of change in the Older People's Programme (Part of Southwark and Lambeth Integrated Care [SLIC]): The King's Fund, 2015.

14. Hospital Episode Statistics. http://content.digital.nhs.uk/hes

15. Bardsley M, Blunt I, Davies S, et al. Is secondary preventive care improving? Observational study of 10-year trends in emergency admissions for conditions amenable to ambulatory care. BMJ Open 2013;3:e002007.

16. Sekhon JS. Multivariate and propensity score matching software with automated balance optimization: the matching package for R. J Stat Softw 2011;42.

17. Wolfe C, Round T, Parkin D, et al. Southwark and Lambeth Integrated Care Programme. Evaluation. Report to the SLIC Sponsor Board: King's College London, 2016.

18. NHS Improvement. National Tariff Payment System $2017 / 18$ and 2018/19. https://improvement.nhs.uk/resources/national-tariff-1719/

19. University of Kent and London School of Economics. Personal Social Services Research Unit Costs of Health and Social Care. 2017. http://www.pssru.ac.uk/pub/uc/uc2017/services.pdf

20. Powell Davies G, Williams AM, Larsen K, et al. Coordinating primary health care: an analysis of the outcomes of a systematic review. Med $J$ Aust 2008;188(8 Suppl):S65-8.

21. Ferris $\mathrm{T}$, Weil $\mathrm{E}$, Meyer $\mathrm{G}$, et al. Cost savings from managing high risk patients. The Healthcare Imperative: Lowering Costs and Improving Outcomes: Workshop Series Summary: National Academies Press, 2010:301-10.

22. Ling T, Brereton L, Conklin A, et al. Barriers and facilitators to integrating care: experiences from the English Integrated Care Pilots. Int J Integr Care 2012;12. 